\title{
La tecnología en la educación: un caso de uso en el ambiente universitario y su impacto en las prácticas de alumnos y docentes
}

\author{
Eduardo Méndez*
}

\begin{abstract}
"Pocas cosas son seguras sobre la enseñanza y el aprendizaje en el siglo XXI. Una de las certezas es que existirán nuevos actores en el proceso educacional y nuevas formas de interacción entre los mismos. A medida que se desarrollan las aulas online, los alumnos, docentes y profesionales asumirán nuevos roles en el aprendizaje y la enseñanza".
\end{abstract}

Cummings, Bonk \& Jacobs (2002)

\section{Resumen}

Este artículo sintetiza y reflexiona sobre dos trabajos de investigación orientados a la descripción e interpretación de las formas de uso e impacto de las nuevas tecnologías en una experiencia del ámbito universitario.

Concretamente el objeto de estudio se centra en las modalidades de uso de un entorno de aprendizaje virtual y su incidencia en las prácticas de docentes y alumnos. Para ello se ha realizado el análisis documental del material publicado, las interacciones y el comportamiento de acceso de usuarios; entrevistas en profundidad a docentes y alumnos, y encuestas a alumnos. Esta estrategia ha permitido generar una visión integral de la experiencia mediante la identificación de categorías de uso y el acercamiento a la comprensión de su impacto.

Marcadas diferencias en los procesos de incorporación y normalización de uso de la tecnología señalan "distintos estadios de adhesión y aprovechamiento" entre los dos actores centrales. La dificultad de los docentes para establecer significatividad y reflexión sobre la recomposición de la práctica de enseñanza a la luz de la innovación, contrasta con la identificación y la adopción intuitiva y natural de los beneficios reconocidos, valorados y reclamados por los estudiantes.

\begin{abstract}
This article considers the results of two research studies into the use of virtual learning environments (VLES) and their impact on learning and teaching in Uruguayan Higher Education. The purpose of these studies was to establish VLE usage patterns by learners and teachers on face-to-face undergraduate and post-graduate programmes and to determine their incidence in terms of teaching practice and learning strategies. Materials published by teachers on ORT Uruguay University's VLE were analysed, as were forum-based interactions and user access logs. Data were also collected via a student survey and in-depth interviews with students and teachers. Both studies into VLE usage -one focusing on students, the other on teachers- provide a holistic view of the experience, including a detailed categorisation of usage patterns. This contributes to furthering our understanding of the impact of VLES on learning and teaching in Higher Education.
\end{abstract}


Significant differences were found, for example, in the processes of technology incorporation and normalisation by learners and teachers. The level of understanding and support of the proposed technology adoption process is varied. Among other findings, the teachers' difficulties to reconfigure their teaching strategies meaningfully in the light of this innovation contrasts with students' almost intuitive adoption of the VLE, and the potential benefits that its use implies.

\section{Contexto - Finalidad}

Las investigaciones están contextualizadas en la Facultad de Administración y Ciencias Sociales de la Universidad ORT. En ella, desde 1999, se está incursionando en modelos educativos en los que el aula presencial y el trabajo online se complementan. La utilización de un "Ambiente de aprendizaje Virtual" ${ }^{1}$ (O'Leary y Ramsden, 2002) se ha incorporado paulatinamente en el quehacer de la facultad y en las prácticas de sus docentes y alumnos.

Si bien, para los docentes, la adopción de esta innovación no fue prescriptiva, su expansión estuvo marcada por procesos de "inducción". Institucionalmente se dispuso del espacio, equipamiento y orientación necesarios para que los actores pudieran desenvolverse en forma adecuada.

La introducción de la tecnología procura la generación de recursos que enriquezcan los procesos de enseñanza y la calidad en las condiciones de estudio y que, a su vez, cumplan con "la condición de estar disponibles en cualquier momento y desde cualquier lugar" (Dringus \& Terrell, 1999 en Clark, 2001).

En términos concretos se proporciona una "herramienta para el gerenciamiento del curso por parte del profesor que facilita la comunicación, la publicación, el acceso a materiales y ejercitaciones, y procesos de actualización" (Armellini \& Gregori ,2001).

En este sentido, el uso de un VLE supone un cambio cultural significativo que genera debate en torno a prácticas y concepciones sobre enseñar y aprender.

Ambos trabajos surgen, entonces, ante la necesidad de determinar en qué forma las nuevas posibilidades se utilizaron, expandieron e impactaron en el ambiente universitario. En consecuencia, la finalidad de los estudios es la mirada e interpretación de esta experiencia en particular y su objeto es el de contribuir a la comprensión del nuevo escenario delineado por la intervención de la tecnología.

\section{Metodología}

Para los dos estudios se ha adoptado un diseño mixto (cuali-cuantitativo) de corte descriptivointerpretativo.

Particularmente comprende:

- la descripción y análisis del uso de funcionalidades de la herramienta en cursos.

- estudio y taxonomización de las propuestas de los docentes.

- la visión de los docentes y alumnos sobre el impacto de la incorporación de la tecnología en sus prácticas.

- el análisis de la actividad de usuarios en el servidor. 
Metodológicamente se ha procurado obtener un abordaje integral a través de la utilización de:

- el estudio de caso y análisis documental en prácticas de los docentes.

- análisis documental en comportamiento de accesos y actividades de usuarios.

- entrevistas en profundidad a docentes y alumnos.

- encuestas a alumnos.

La población estudiada está integrada por docentes y estudiantes a partir del segundo semestre $^{2}$ en las carreras de grado. Las entrevistas se realizaron en base a criterios de selección intencional. Las encuestas incluyeron muestras representativas de las distintas carreras y niveles de aprendizaje.

\section{La tecnología y los docentes}

La incorporación de las nuevas tecnologías al escenario educativo despierta, al decir de Maggio, un nuevo tiempo, "un tiempo de reconceptualización" (Litwin, 1995), teñido de desafío y compromiso.

No obstante, en la experiencia estudiada y desde la perspectiva docente, no todos los actores adoptan, o se ven tentados a incursionar, en las posibilidades ofrecidas por el nuevo escenario. Por el contrario, la evidencia muestra dificultad a la hora de reconsiderar y modificar las prácticas de enseñanza a la luz de la inclusión de la tecnología. Ésta, que "siempre camina dos pasos más adelante ...que su tratamiento didáctico" (Litwin, 2002), parece, en cierto momento, identificarse más con el concepto de obstáculo que con el de oportunidad de mejora o innovación.

En la mayoría de los casos la incorporación de la tecnología en el quehacer del docente se realizó de manera "efectiva" ${ }^{3}$; en otras palabras, a través de la inducción institucional.

Sin embargo, a pesar del carácter "efectivo" de la incorporación -así como de cierta reticencia inicial- una vez que el docente toma contacto con las nuevas posibilidades reconoce impactos positivos. Este proceso de descubrimiento se produce en forma paulatina, y la profundidad con la que se presenta lauda la modalidad de uso que, en la mayoría de los casos, hace a actividades no propias de la enseñanza.

\section{I dentificando prácticas}

Los casos estudiados muestran que las prácticas de uso de la tecnología -con un propósito casi siempre no explicitado- pueden describirse a través de un continuo. En sus extremos se identifican propuestas claramente definidas.

Por un lado, y en su expresión más simple de uso, distinguimos prácticas donde la herramienta se considera como un sistema de distribución de material y adopta un rol sucedáneo a los repartidos que se deja a disposición de los estudiantes en bedelía o fotocopiadora. En este tipo de proposición, el papel de la herramienta es asimilable al de una "estantería" donde se dispone el material en forma ordenada para que sea retirado. La nueva tecnología se utiliza como medio de implementación de actividades que no hacen a la esencia del enseñar y del aprender. 
En el otro extremo -mucho más complejo y rico- ubicamos propuestas donde el "ambiente virtual" es concebido como extensión de la práctica docente. En otras palabras, la presentación de saberes, la reflexión y las interacciones trascienden el salón de clase. Las prácticas de uso de la tecnología se muestran como un componente sustantivo del escenario de enseñanza. Los casos de mayor destaque adoptan concepciones propias de la educación a distancia. Evidencian características de material "autocontenido" formulado con criterios de "anticipación" y pensando en la "ausencia del docente", esto es, para lugares y tiempos distintos del educando.

En estas propuestas se da una marcada preocupación por la mediación tecnológica en el proceso de interacción alumno-contenido. Son desplegadas estrategias orientadoras que ofrecen al estudiante un permanente marco de referencia respecto a la significatividad y contextualización de los conceptos. Fundamentalmente se adoptan construcciones no lineales donde el alumno recorre los componentes relevantes de la materia de acuerdo a sus propios constructos, necesidades e intereses.

Particularmente se ha operado una fuerte personalización del ambiente en función de las estrategias de presentación de información y contenidos. Se evidencia que en estas propuestas el uso de la tecnología se encuentra subordinado a la intención del docente. El valor expresado tanto por la práctica, como por el discurso del docente, radica en las posibilidades aportadas y no en la tecnología en sí misma. Este aspecto, en extremo relevante, muestra comprensión del nuevo escenario y su impacto en el plano didáctico y comunicacional. Da cuenta también de otro hecho mucho más profundo: la reflexión y modificación de las prácticas de enseñanza.

Otro rasgo distintivo de estas propuestas corresponde al sentido de "humanización". En la interacción sitio-contenidos-alumnos se evidencian permanentes "signos de presencia y preocupación" del docente que proporcionan calidez, orientación y "anclaje" a los alumnos.

El cuidado y la responsabilidad con que la tecnología fue incorporada impactan directamente en la calidad de la propuesta de enseñanza, permitiendo inferir el compromiso de los docentes con su práctica. Ésta es considerada como un todo donde lo presencial y lo virtual se articulan.

Una tercera categoría de uso se configura por aquellas materias que se ubican en zonas intermedias del continuo. Son propuestas en las que el ambiente trasciende la concepción de sistema de distribución, pero que no pueden considerarse como extensión del escenario de enseñanza.

En esta categoría los docentes perciben el impacto de la incorporación de la tecnología en términos prácticos. El escenario virtual es utilizado para estructurar, organizar y sistematizar el curso. Los espacios de enseñanza y reflexión se gestan en el salón de clases. La tecnología es utilizada para proporcionar información y actividades online a través de un esquema bidireccional (alumno-docente).

La mayoría de las propuestas estudiadas se ubican en el primer extremo y zonas intermedias del continuo, es decir, se orientan hacia modalidades de uso que no pueden ser consideradas como parte de la práctica de enseñanza.

\section{Las dificultades en la utilización de la tecnología: ¿un problema de significatividad?}

La escasez de propuestas donde la tecnología se integra a la enseñanza nos provoca interrogarnos: ¿la dificultad está vinculada a la práctica de enseñanza, a la tecnología en sí misma o a otros factores?

\section{8 - Universidad ORT Uruguay}


Inicialmente se reconocen factores casi "evidentes" que operan como inhibidores en la adopción de la tecnología al quehacer docente.

Uno de ellos es la percepción de la tecnología como importante "consumidora" de tiempo. La dedicación temporal que insume su utilización se manifiesta desde dos dimensiones. Por un lado, el tiempo necesario para la elaboración, edición y actualización sistemática de material; por otro, el tiempo requerido por las comunicaciones durante y después del dictado del curso. Las funcionalidades consumen tiempo en una forma más o menos constante al nivel de dedicación. El perfil y la extracción del docente determinan que, justamente, el recurso tiempo sea extremadamente escaso.

Otra dificultad percibida refiere a la tecnología en sí misma y especialmente a la falta de transparencia hacia los actores. Comprende aspectos tales como la amigabilidad con el usuario, la facilidad de uso de las distintas funcionalidades, la comprensión del ambiente y las posibilidades que brinda. Adicionalmente "muchos alumnos se encuentran 'por delante' de sus docentes en términos de fluidez informática y familiaridad con las nuevas tecnologías" (O'Leary y Ramsden, 2002). Enfrentar esta asimetría a "favor" de los estudiantes no es un aspecto menor.

Por lo general el docente "aborda en soledad" (Grünberg, 2000) las cuestiones vinculadas a su práctica. La carencia de espacios o actitudes de trabajo colaborativo constituye otro obstáculo a la hora de incorporar la tecnología. Su uso, generalmente, se construye en un proceso de aproximaciones sucesivas que transita por instancias personales de ensayo y error.

Algunos docentes delegan en la dimensión institucional la definición de posiciones y el establecimiento de planes de acción sobre la incorporación y adopción de la tecnología educativa. En otras palabras, algunos actores abren un tiempo de espera para que desde lo institucional se les proporcione un "andamio" que oriente el cambio cultural estableciendo límites, aspiraciones y recursos.

Sin embargo estos aspectos no explican las limitantes o inhibidores en aquellos docentes que son reconocidos por sus "buenas prácticas de enseñanza" y que en cierta forma tenían resueltos los dilemas y obstáculos mencionados. Se trató de determinar qué "otro factor" inhibía en estos casos el uso de la tecnología o la incorporación de la misma a su acto de enseñanza.

Su taxonomización mostró que el énfasis se puso en la publicación de ciertos contenidos presentando recorte hacia el resto de las funcionalidades. Este hecho no es fortuito. El accionar y discurso de los docentes, refleja la relevancia y preeminencia que, en el "ambiente de aprendizaje virtual" utilizado, se le da a la dimensión publicación de materiales. Indudablemente el docente integra cambios en la medida que suponga significatividad para su práctica. En el proceso de incorporación de la tecnología, estos docentes han subordinado la significatividad a la lógica impuesta por la propia tecnología y no a la reflexión y recomposición de su práctica.

\section{La tecnología y los alumnos}

La relación de los estudiantes con la tecnología nos muestra literalmente la otra cara de la moneda. En comparación con los docentes -y desde la perspectiva de usuario- los alumnos no muestran ambigüedades o dudas en su utilización. En forma natural han valorado e incorporado los aportes que el nuevo escenario les ha brindado en su vida como estudiantes. 
Seguramente, ni la hipótesis más positiva, hubiera concebido al 90\% de los alumnos efectuando la enfática afirmación de que "todas las materias debieran tener sus contenidos en forma virtual".

El alumno identifica -desde un juicio crítico y racional- los beneficios aportados por el proceso de innovación: la tecnología es un instrumento sin el cual "se puede estudiar", pero con el que "decididamente se estudia mucho mejor".

Su incorporación, aunque condicionada y limitada, mejoró, incluyó, apoyó e integró alumnos presenciales "típicos" con alumnos "no tradicionales", "estudiantes profesionales" con "estudiantes-trabajadores". Esto determina que el uso de la tecnología sea percibido como un aporte clave en la generación de una comunidad académica abierta y diversa, que proporciona una sensación de pertenencia más allá de edificios, salones y nombres.

\section{¿Una experiencia restringida?}

El propósito de uso generalmente adoptado por los docentes determina que predomine la concepción de "estantería". La distribución de materiales a través de un medio práctico y confiable se constituye como la principal aplicación identificada por la mayoría de los estudiantes. Esta finalidad se estima limitada.

La disposición y difusión de uso -por parte del docente- son los factores que marcan la diferencia a la hora de poner en marcha el uso de la tecnología en el escenario educativo. En otros términos diríamos que, desde la perspectiva de los estudiantes, el aspecto clave en el desarrollo de buenas prácticas en la utilización de la tecnología pasa por el proceso de normalización del propio docente.

La diferenciación entre las distintas propuestas se hace evidente. Aquellos docentes que adoptaron una estrategia de evolución continua, enriqueciendo los primeros contenidos, explicitando objetivos y explotando la interacción son diferenciados mediante la evidencia de los accesos y la crítica evaluación de los estudiantes. Éstos resaltan, en especial, a aquellos docentes que a través de sus prácticas permitieron a los alumnos ser "responsables de su propio involucramiento en las tareas...en la toma de direcciones, interacción con los recursos y resolución de problemas" (Lander, 1997).

La diferencia en concepciones de uso por parte de los docentes, junto con la ausencia de propuestas virtuales en algunas de las materias son aspectos poco comprendidos -y no del todo aceptados- por los estudiantes. Desde su perspectiva, y en un proceso de franca normalización del uso de la tecnología, reclaman un "piso", un estadio mínimo necesario.

La consideración del uso de la tecnología instala en la visión de los estudiantes la reflexión sobre las prácticas de los docentes.

\section{El lugar de la mirada de los estudiantes}

La mirada de los estudiantes hacia las prácticas de uso de la tecnología por los docentes se lauda desde una visión realista. No caen en un facilismo esperando que se les resuelva su vida como alumnos. Esto es, no se pide todo "digerido", se asume que existen otras fuentes, que el éxito en su vida de estudiantes pasa por varios carriles, entre los que, la tecnología constituye 
un apoyo valioso.

En un escenario de enseñanza privado, donde la disciplina impartida proviene del campo de la administración y la economía, no es de extrañar que el alumno se identifique a sí mismo como estudiante a la vez que como cliente. Este cliente, crítico y racional, a la hora de evaluar demanda "un producto" que lo satisfizo y que, fundamentalmente, le es útil. "Que todos estén", "que si está se use" y "emparejar para arriba" son expresiones que definen lo expresado. Paradójicamente la presencia de estos "reclamos" -de aparente connotación negativa- da cuenta del profundo efecto de la tecnología en la vida de los estudiantes.

La sustitución de la fotocopiadora por una "estantería virtual" no alcanza para explicar este impacto presente -en forma independiente a la carrera o semestre- en la opinión de los alumnos. La utilización de la tecnología ha trascendido el umbral marcado por la práctica instaurada en la generalidad de las materias.

La actitud innovadora de algunos docentes, donde la incorporación de la tecnología es parte de su ejercicio de enseñanza, genera en la percepción de los estudiantes la noción de "prácticas deseables" y convenientes. Ante ello, la afirmación de "coloco los materiales en un VLE, por lo tanto mis alumnos hacen e-learning" se torna obsoleta. La necesidad de establecer procesos comunicacionales efectivos y focalizados, determinar clara y explícitamente la propuesta de utilización de la tecnología y desarrollar contenidos desde una perspectiva diferente a la del material presentado de apoyo en clase, determinan la clave para lograr un efectivo apoyo al proceso de aprendizaje.

Los alumnos han sido capaces de diferenciar este concepto estableciendo -desde juicios objetivos y certeros- cuáles han sido las propuestas que mayor impacto han tenido en su vida como estudiantes. Visualizan la necesidad de tangibilizar aquellos aspectos que se identifican como potencialmente beneficiosos y deseables, extendiendo "las buenas prácticas virtualizadoras" a todas las materias.

\section{Conclusiones}

Nuestros estudios reconocen, describen e intentan acercar comprensión respecto al uso de la tecnología en una experiencia de incorporación en el ámbito universitario.

Desde la dimensión docente se identifican fundamentalmente dos categorías de uso. En primer lugar -y en su concepción más generalizada- se ubican aquellas prácticas que no hacen a la esencia de la enseñanza. Aquí la tecnología oficia de apoyo al propio docente en su actividad, organizando, respaldando y distribuyendo los materiales del curso. En general, la significatividad aparece subordinada a la lógica impuesta por las propias herramientas tecnológicas y no a la reflexión y recomposición de la práctica.

Por otro lado aparecen aquellas proposiciones en las que, a través de procesos de innovación permanente, los docentes plantean prácticas vinculadas a la enseñanza. La extensión del ámbito de presentación de saberes define esta categoría. El uso de la tecnología se subordina a la intención del docente. Éste considera su práctica como un todo articulando su propuesta presencial y virtual.

Los alumnos aparecen como los usuarios por excelencia. En un proceso de franca normalización en el uso -que se da por igual en todas las carreras y grados de avance- reconocen 
e incorporan todos aquellos aspectos positivos vinculados a la tecnología.

Desde una visión crítica y realista identifican y valoran las distintas propuestas desplegadas por los docentes. La tecnología instala la mirada del alumno hacia la práctica del docente y fija su expectativa de acuerdo con las mejores prácticas desplegadas. La ausencia de la práctica virtualizadora en algunas de las materias es percibida como seria carencia.

La introducción de la tecnología delinea, entonces, un escenario caracterizado por distintos estadios de adhesión, aprovechamiento y significatividad entre los dos actores centrales: docentes y alumnos.

En consecuencia, se instala el reto de ahondar los impactos positivos en la vida de los estudiantes a través de un salto cualitativo que explote y difunda el uso de las fortalezas de la tecnología en beneficio de la calidad de la enseñanza.

La evolución desde un esquema caracterizado por la integración de herramientas online al curso presencial a un escenario donde el curso, como unidad, hace uso de entornos presenciales y entornos no presenciales -ambos igualmente importantes y centrales- marca, en el uso de la tecnología, el camino hacia "la generación de genuinos y significativos ambientes de aprendizaje» (Lorenzo, 2000).

\section{Bibliografía y notas:}

Armellini \& Gregori. 2001. Criterios para la incorporación efectiva de internet como apoyo a cursos presenciales. Informe de Investigación. Montevideo, Universidad ORT.

Cummings, Bonk \& Jacobs. 2002. Options for online communication and interactivity. Internet and Higher Education, № 5.

Grünberg, J. 2000. Teachers and computer-mediated communication: a study of the development of collegiality among secondary school teachers using electronic mail. Tesis Doctoral, University of Oxford.

Lander, D. 1997. Online Teaching: Educational Considerations, Royal Melbourne Institute of Technology. (online) (citado octubre 2004). Disponible en internet:<http:// homepages.eu.rmit.edu.au/ resdl/teaching3.html>.

Litwin, E. 2002. Las nuevas tecnologías y las prácticas de la enseñanza en la universidad (online) (citado octubre 2004). Disponible en internet:< http://www.litwin.com.ar>.

Litwin, E. 1995. Tecnología Educativa: Política, historias, propuestas. Buenos Aires: Paidós.

Lorenzo, E. 2000. Enseñanza a distancia y educación a distancia: ¿de qué estamos hablando? Cuadernos de Investigación Educativa Vol. 1 No. 6 julio 2000. Montevideo: 
Universidad ORT Uruguay.

O'Leary, R. \& Ramsden, A. 2002. Virtual learning environments. University of Bristol.

(online) (citado octubre 2004). Disponible en internet: <http://www.economics.Itsn.ac.uk/ handbook/vle/s.

1 "VLEs" o "LMSs" por sus siglas en inglés.

${ }^{2}$ La decisión de excluir el primer semestre se basa en el necesario contacto y familiaridad de los alumnos con la tecnología.

${ }^{3}$ Término acuñado por Maggio.

* Master en Educación (Cand.), Universidad ORT. Profesor de Dibujo, Instituto de Profesores Artigas. 